\title{
Insm1a Regulates Motor Neuron Development in Zebrafish
}

\begin{abstract}
Jie Gong ${ }^{1 \dagger}$, Xin Wang ${ }^{2 \dagger}$, Chenwen Zhu ${ }^{2}$, Xiaohua Dong ${ }^{3}$, Qinxin Zhang ${ }^{3}$, Xiaoning Wang ${ }^{2}$, Xuchu Duan ${ }^{1}$, Fuping Qian ${ }^{2,4}$, Yunwei Shi ${ }^{2}$, Yu Gao ${ }^{2}$, Qingshun Zhao ${ }^{3 *}$, Renjie Chai ${ }^{2,4 *}$ and Dong Liu ${ }^{2 *}$

${ }^{1}$ School of Life Science, Nantong University, Nantong, China, ${ }^{2}$ Co-innovation Center of Neuroregeneration, Key Laboratory of Neuroregeneration of Jiangsu and Ministry of Education, Nantong University, Nantong, China, ${ }^{3}$ MOE Key Laboratory of Model Animal for Disease Study, Model Animal Research Center, Nanjing University, Nanjing, China, ${ }^{4}$ Key Laboratory for Developmental Genes and Human Disease, Ministry of Education, Institute of Life Sciences, Southeast University, Nanjing, China
\end{abstract}

Insulinoma-associated1a (insm1a) is a zinc-finger transcription factor playing a series of functions in cell formation and differentiation of vertebrate central and peripheral nervous systems and neuroendocrine system. However, its roles on the development of motor neuron have still remained uncovered. Here, we provided evidences that insm1a was a vital regulator of motor neuron development, and provided a mechanistic understanding

OPEN ACCESS

Edited by:

Chen Zhang

Peking University, China

Reviewed by:

Zhiqiang Yan,

Fudan University, China

Jie He,

Institute of Neuroscience, Shanghai

Institutes for Biological Sciences

(CAS), China

${ }^{*}$ Correspondence:

Qingshun Zhao

qingshun@nju.edu.cn

Renjie Chai

renjiec@seu.edu.cn

Dong Liu

livdongtom@gmail.com

${ }^{\dagger}$ These authors have contributed equally to this work.

Received: 01 July 2017 Accepted: 14 August 2017 Published: 28 August 2017

Citation:

Gong J, Wang $X$, Zhu C, Dong X Zhang Q, Wang X, Duan X, Qian F, Shi Y, Gao Y, Zhao Q, Chai R and Liu D (2017) Insm1a Regulates Motor Neuron Development in Zebrafish.

Front. Mol. Neurosci. 10:274 doi: 10.3389/fnmol.2017.00274 of how it contributes to this process. Firstly, we showed the localization of insm1a in spinal cord, and primary motor neurons (PMNs) of zebrafish embryos by in situ hybridization, and imaging analysis of transgenic reporter line Tg(insm1a: mCherry) ${ }^{\text {ntu805 }}$. Then we demonstrated that the deficiency of insm1a in zebrafish larvae lead to the defects of PMNs development, including the reduction of caudal primary motor neurons (CaP), and middle primary motor neurons (MiP), the excessive branching of motor axons, and the disorganized distance between adjacent CaPs. Additionally, knockout of insm 1 impaired motor neuron differentiation in the spinal cord. Locomotion analysis showed that swimming activity was significantly reduced in the insm1a-null zebrafish. Furthermore, we showed that the insm1a loss of function significantly decreased the transcript levels of both olig2 and $n k \times 6.1$. Microinjection of olig2 and $n k x 6.1$ mRNA rescued the motor neuron defects in insm1a deficient embryos. Taken together, these data indicated that insm1a regulated the motor neuron development, at least in part, through modulation of the expressions of olig2 and $n k \times 6.1$.

Keywords: insm1a, motor neuron, differentiation, zebrafish, development

\section{INTRODUCTION}

In vertebrates, motor neurons have precise subtype identities that characterized by a number of morphological criteria, such as soma location, and shape, axon path, and target muscle innervation (Shirasaki and Pfaff, 2002; Lewis and Eisen, 2003). Meanwhile, motor neurons generally extend their axonal trajectory with a highly stereotyped manner during the nervous system development (Eisen, 1991; Palaisa and Granato, 2007). It has been reported that in chick and bullfrog, their motor neuron axons always followed the conservative pathways in order to project to appropriate regions of target musculatures (Landmesser, 1980; Farel and Bemelmans, 1985). In the embryo and larva of zebrafish, there are two different kinds of spinal motor neurons, which are called primary 
motor neurons (PMNs), and secondary motor neuron (SMNs) (Myers, 1985; Myers et al., 1986). The PMNs can be further classified into three groups, caudal primary motor neurons $(\mathrm{CaP})$, middle primary motor neurons $(\mathrm{MiP})$, and rostral primary motor neurons (RoP), by the positions of somata in the spinal cord, and the trajectory of neuron axons (Myers et al., 1986; Westerfield et al., 1986). CaPs, whose somata locate in the middle of each spinal cord hemisegment, can innervate ventral axial muscle, and have been well-studied because of their easy observation and distinct axon projection (Myers et al., 1986; Rodino-Klapac and Beattie, 2004). MiPs project axons to innervate the dorsal axial muscle, while RoPs project axons to control the middle muscle (Rodino-Klapac and Beattie, 2004). Although the somata of the three identifiable PMNs are localized in different position in the spinal cord, their axons pioneer to the myoseptum through a mutual exit point (Eisen et al., 1986). Due to the identifiability of the three kinds of PMNs, they have already become an excellent system to study motor axon guidance and their intraspinal navigation (Beattie et al., 2002).

The insulinoma-associated 1 (insm 1) gene, which is first isolated from an subtraction cDNA library of insulinoma tumor cells, encodes a DNA-binding zinc finger transcription factor with SNAG repressor motifs in N-terminal as well as Cys2His2 $\mathrm{Zn}$ finger motifs in C-terminal, and widely expresses in the developing nervous system, endocrine cells, pancreatic cells, and related neuroendocrine tumor cells (Goto et al., 1992; Xie et al., 2002; Jacob et al., 2009; Lan and Breslin, 2009; Jia et al., 2015b). Consequently, extensive studies focused on the biological function of insm 1 in nervous, and endocrine cell proliferation, differentiation, and transformation have been reported in the model organisms (Farkas et al., 2008; Wildner et al., 2008; Lan and Breslin, 2009; Ramachandran et al., 2012; Jia et al., 2015a,b). For example, in the insm 1 knockout mice, its endocrine progenitor in the developing pancreas were less differentiated, meanwhile hormone production, and cell migration also exhibited seriously defects (Osipovich et al., 2014). Farkas et al. reported that compared to the wild type and heterozygous mice, the number of basal progenitors in the insm1 null dorsal telencephalon ( $\mathrm{dTel}$ ) was decreased almost half, and the radial thickness of dTel cortical plate as well as the neurogenesis in the neocortex were also predominantly reduced after lacking insm1 gene (Farkas et al., 2008). In the zebrafish, insm 1 a can regulate a series of related genes, which are necessary for the Müller glia (MG) formation, and differentiation as well as the zone definition of injury-responsive MG to participate in the retina regeneration (Ramachandran et al., 2012). Moreover, it was also reported that during the development of zebrafish retina, insm 1 could regulate cell cycle kinetics and differentiation of the progenitor cells by acting the upstream of the basic helix-loop-helix (bHLH) transcription factors, and the photoreceptor specification genes (Forbes-Osborne et al., 2013). Although insm1a is widely detected in the nervous system and its necessity in the brain and retina development have been also illuminated well, little is known about the function and molecular mechanisms of insm 1a on the formation and development of other neuronal types, especially in the zebrafish.
The zebrafish has become an excellent model system to investigate the mechanisms of the neuron formation and its axonal pathfinding due to the accessible observation of motor neurons from the initial stages of embryo development (Zelenchuk and Bruses, 2011). Here, we examined the function of insm $1 a$ in the primary motor neurons development by CRISPR/ Cas9-mediated knockout in the $\operatorname{Tg}(m n x 1: G F P)^{m / 2}$ transgenic zebrafish and investigated the possible transcriptional network during this process.

\section{MATERIALS AND METHODS}

\section{Zebrafish Line and Breeding}

The zebrafish embryos and adults were maintained in zebrafish Center of Nantong University under conditions in accordance with our previous protocols ( $\mathrm{Xu}$ et al., 2014; Wang et al., 2016). The transgenic zebrafish line, $\operatorname{Tg}(m n x 1: G F P)^{m l 2}$, has been described in the previous work (Zelenchuk and Bruses, 2011).

\section{RNA Isolation, Reverse Transcription and Quantitative PCR}

Total RNA was extracted from zebrafish embryos by TRIzol reagent according to the manufacturer's instructions (Invitrogen, USA). Genomic contaminations were removed by DNaseI, and then $2 \mu \mathrm{g}$ total RNA was reversely transcribed using a reversed first strand cDNA synthesis kit (Fermentas, USA) and stored at $-20^{\circ} \mathrm{C}$. qRT-PCR was performed using the corresponding primers (Supplementary Table 1 ) in a $20 \mu \mathrm{l}$ reaction volume with $10 \mu \mathrm{l}$ SYBR premix (Takara, Japan) and elongation factor $1 a$ (efla) was used as the internal control. All samples were analyzed in triplicate.

\section{Whole Mount In situ Hybridization}

A 501 bp cDNA fragment of insm1a was amplified from the cDNA library that established from wild type (WT) $\mathrm{AB}$ embryos using the specific primers of insm1a F1 and insm1a R1 (Supplementary Table 1). Digoxigenin-labeled sense and antisense probes were synthesized using linearized pGEM-Teasy vector subcloned with this insm1a fragment by in vitro transcription with DIG-RNA labeling Kit (Roche, Switzerland). Zebrafish embryos and larvae were collected and fixed with $4 \%$ paraformaldehyde (PFA) in phosphate-buffered saline (PBS) for one night. The fixed samples were then dehydrated through a series of increasing concentrations of methanol and stored at $-20^{\circ} \mathrm{C}$ in $100 \%$ methanol eventually. Whole mount in situ hybridization was subsequently performed as described in the previous study (Huang et al., 2013).

\section{Establishment of Tg(insm1a: EGFP) ${ }^{\text {ntu804 }}$ and Tg(insm1a: mCherry) ${ }^{\text {th }} 805$ Transgenic Line}

Transgenic zebrafish were created using the Tol2kit transgenesis system and Gateway vectors. The insmla promoter was cloned and insert into the p5E-MCS entry vector. A multiSite Gateway vector construction reaction (Invitrogen, USA) was conducted with the resulting p5E-insm1a together with pMEEGFP (or mCherry) and p3E-polyA subcloned into the 
pDestTol2pA2 to produce insm1a: EGFP or insm1a: mCherry construct. Subsequently, this construct was co-injected with tol2-transposase mRNAs into zebrafish one to two-cell-stage embryos to create the $\operatorname{Tg}(\text { insm1a: EGFP) })^{n t u 804}$ and $\operatorname{Tg}($ insm1a: $m$ Cherry) ${ }^{n t u 805}$ transgenic line.

\section{sgRNA/ Cas9 mRNA Synthesis and Injection}

Cas9 mRNA was obtained by in vitro transcription with the linearized plasmid pXT7-Cas9 according to the procedure previously described. The sgRNAs were transcribed from the DNA templates that amplified by PCR with a pT7 plasmid as the template, a specific forward primer and a universal reverse primer (Supplementary Table 1) (Chang et al., 2013; Qi et al., 2016). Transgenic zebrafish lines $\operatorname{Tg}(m n x 1: G F P)^{m l 2}$, were natural mated to obtain embryos for microinjection. One to two-cell stage zebrafish embryos were injected with $2-3 \mathrm{nl}$ of a solution containing $250 \mathrm{ng} / \mu \mathrm{l}$ Cas $9 \mathrm{mRNA}$ and $15 \mathrm{ng} / \mu \mathrm{l}$ sgRNA. At $24 \mathrm{~h}$ post fertilization (hpf), zebrafish embryos were randomly sampled for genomic DNA extraction according to the previous methods to determine the indel mutations by sequencing.

\section{Morpholino and mRNAs Injections}

Translation blocking antisense Morpholino (MOs; Gene Tools) against the ATG-containing sequence was designed (5'-AAA TCCTCTGGGCATCTTCGCCAGC- $3^{\prime}$ ) to target the translation start site according to the manufacturer's instruction and the other MO oligo (5'-CCTCTTACCTCAGTTACAATTTATA-3') was used as standard control. The MOs were diluted to 0.3 $\mathrm{mM}$ with RNase-free water and injected into the yolk of one to two-cell stage embryos and then raised in E3 medium at $28.5^{\circ} \mathrm{C}$.

The cDNAs containing the open reading frame of the target genes were cloned into $\mathrm{PCS}^{+}$vector respectively and then were transcribed in vitro using the mMESSAGE mMACHIN Kit (Ambion, USA) after the recombinant plasmids linearized with NotI Restriction Enzyme (NEB, England), and then the capped mRNAs were purified by RNeasy Mini Kit (Qiagen, Germany). 2 $\mathrm{nl}$ target genes and mCherry mRNA mixture (1:1) were injected at $20 \mathrm{ng} / \mu \mathrm{l}$ into $1 / 2$-cell stage embryos.

\section{Locomotion Analysis in Zebrafish Larvae}

To determine whether the deficiency of insmla affect spontaneous movement, knockout, and normal larvae were raised in a 24-well-culture plate with one larva in each wellfilled with $1 \mathrm{ml}$ E3 medium. The 24-well-culture plate was transferred to the Zebralab Video-Track system (Zebrabox, France) equipped with a sealed opaque plastic box that kept insulated from laboratory environment, an infrared filter and a monochrome camera. After adapting for $30 \mathrm{~min}$, traveled distances of the larvae were videotaped with every 5 mins forming a movement distance and trajectory by the linked software.

\section{Microscopy and Statistical Analysis}

Zebrafish embryos were anesthetized with E3/0.16 mg/mL tricaine/1\% 1-phenyl-2-thiourea (Sigma, USA) and embedded in $0.8 \%$ low melt agarose, and then were examined with a Leica
TCS-SP5 LSM confocal imaging system. For the results of in situ hybridization, Photographs were taken using an Olympus stereomicroscope MVX10. Statistical comparisons of the data were carried out by student's $t$-test or one-way analysis of variance (ANOVA) followed by Duncan's test, and $P<0.05$ were considered statistically significant. All statistical analysis was performed using the SPSS 13.0 software (SPSS, USA).

\section{RESULT}

\section{Insm1a is Expressed in Spinal Cord and PMNs of Zebrafish}

To analyze the expression of insm1a in zebrafish nervous system, we performed the whole amount in situ hybridization (WISH) analysis with a digoxigenin-labeled insm1a probe. Similar to the previous study (Lukowski et al., 2006), at late somitogenesis (24 hpf) insm 1a transcripts were apparently localized in ventral part of the neurons in the spinal cord, where most of the motor neurons located at this stage (Figure 1A).

To further determine the localization of insm1a, we generated the $\operatorname{Tg}$ (insm1a: EGFP) $)^{\text {ntu804 }}$ and $\operatorname{Tg}(\text { insm1a: mCherry })^{n t u 805}$ transgenic zebrafish lines, in which the insmla promoter directed expression of EGFP or mCherry respectively. It was shown that at $30 \mathrm{hpf}$ the insm1a:mCherry and insm1a:EGFP expression was observed in the spinal cord, retina and brain, which was similar with the results of in situ hybridization (Figures 1B,B'; Supplementary Figures 1A,A') (Lukowski et al., 2006). In addition, we found that insm1a:EGFP expression was highly activated in Müller glia of injury sites in adult zebrafish retina (Supplementary Figure 1B), which was consistent with the ISH data carried out by other researchers (Ramachandran et al., 2012). These results suggested that the transgenes recapitulated the endogenous insm1a expression.

To investigate whether insm 1a is expressed in motor neurons, we outcrossed $\mathrm{Tg}$ (insm1a: $m$ Cherry) (ntu 805 transgenic line with $\operatorname{Tg}(m n x 1: G F P)^{m l 2}$ line, in which motor neurons were labeled with GFP (Zelenchuk and Bruses, 2011). We found that the GFP ${ }^{+}$ motor neurons were also labeled with mCherry fluorescence (Figures 1C-C"), suggesting insm1a was expressed in motor neurons.

\section{Knockout of insm1a Caused Primary Motor Neurons Developmental Defect}

In order to examine whether insmla is required for the development of motor neuron, the CRISPR/Cas9 system was utilized to knockout insm1a in $\operatorname{Tg}(m n x 1: G F P)^{m l 2}$ transgenic zebrafish line. To ensure complete disruption of functional proteins, we chose the target sites near the translation start codon (ATG) in the exon1 of zebrafish insm 1a (Supplementary Figure 2A). The selected gRNA-Cas9 system efficiently induced mutations in the targeting site with four types of mutations were identified (Supplementary Figure 2B). The mutated alleles included a 5-bp deletion, an 8-bp deletion and two 10-bp deletions, which all resulted in reading frame shift and premature translation termination (Supplementary Figure 2C). In addition, 

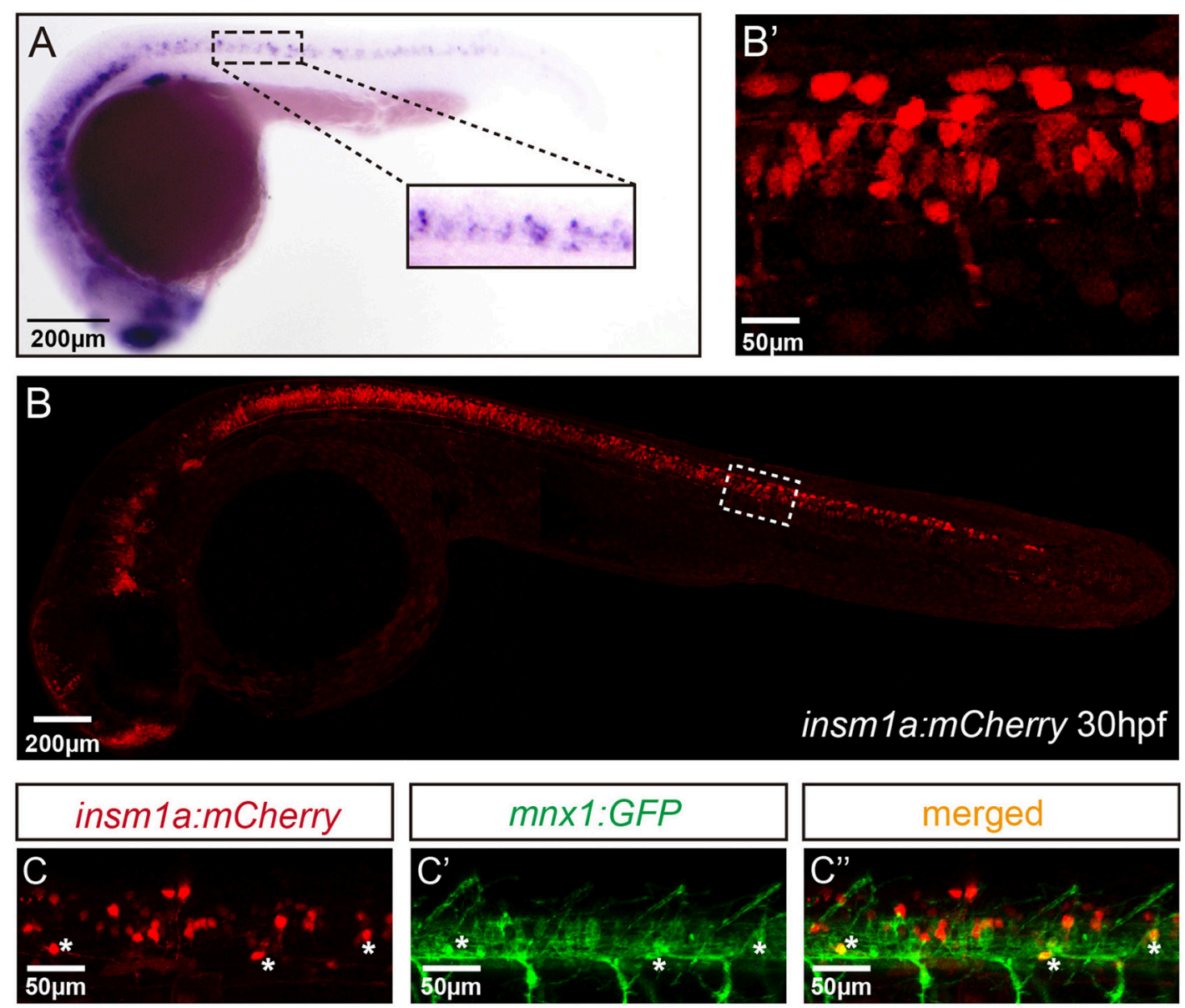

FIGURE 1 | Insm1a expression in embryonic zebrafish spinal cord and primary motor neurons. (A) At 24 hpf, the in situ hybridization signal of insm1a is localized in the spinal cord., Scale bar $=200 \mu \mathrm{m}$. (B) The confocal imaging analysis of the transgene insm1a:mCherry expression at 30 hpf. Square in dash line indicates the magnified region in (B') Scale bar $=50 \mu \mathrm{m}$. (C,C',C'). Confocal imaging analysis of Tg(mnx1:GFP)m/2 $\times$ Tg(insm1a: mCherry) ${ }^{\text {ntu805 }}$ transgenic line.

these lines showed the same phenotypes and the 8-bp deletion mutant line was used for the following experiments.

It was observed that insmla knockout caused obvious developmental defect of motor neurons (Figure 2). Firstly, the number of MiPs and $\mathrm{CaPs}$ were significantly reduced in the insm1a mutants (Figure 2A). We counted the number of Caps and classified the zebrafish embryo into three categories by its defective degree: severe group with over $80 \%$ loss of Caps, moderate group with $<80 \%$ loss and normal group with $<20 \%$ loss (In the following statistical analysis, the zebrafish with $<20 \%$ loss was defined as normal, whereas, it was abnormal). These results revealed that $48.1 \%$ severe and $32.1 \%$ moderate defect were found in the insm 1 a mutants, while there was only $7.9 \%$ moderate defect in the control group (Figure 2B). Similarly, the MiPs were also obviously impaired in insm 1a knockout embryos (Figures 2A,F). Importantly, we found that these abnormal phenotypes of motor neurons could not recover at later stages we checked (Supplementary Figure 3).

Moreover, the morphology of motor neurons was significantly affected in the insm1a mutants (Figures 2A,D,E). The axons of
Caps in insm1a mutants were shorter and failed to reach the ventral musculatures. The branches density of the Caps in insm1a mutants was higher than that in control. For example, in the insm1a mutants, there were around 34 branch points of per $1 \mathrm{~mm} \mathrm{CaP}$ axon at $48 \mathrm{hpf}$, while only 31 in control embryos (Figure 2E). With the larvae development, the excess branching became more, and more pronounced (Supplementary Figure 3). In addition, statistical analysis revealed that the average length of each $\mathrm{CaP}$ anon in the insm1a mutants was $707.9 \mu \mathrm{m}$ at $48 \mathrm{hpf}$, while in control embryos it increased to $1367.9 \mu \mathrm{m}$ (Figure 2D). Interestingly, we also found that the distances between adjacent CaPs were significantly variant in insm 1a mutants (Figure 2C).

In order to validate the developmental defects of motor neuron was specifically caused by the insm1a inactivation, further experiments were carried out. The embryos that injected with an insm1a translation blocking morpholino displayed the similar motor neuron with that observed in the insm1a mutants (Supplementary Figure 4). To confirm phenotypic specificity induced by the insmla MO injection, we performed rescue experiment by co-injection of $50 \mathrm{ng}$ of insm1a mRNA with 

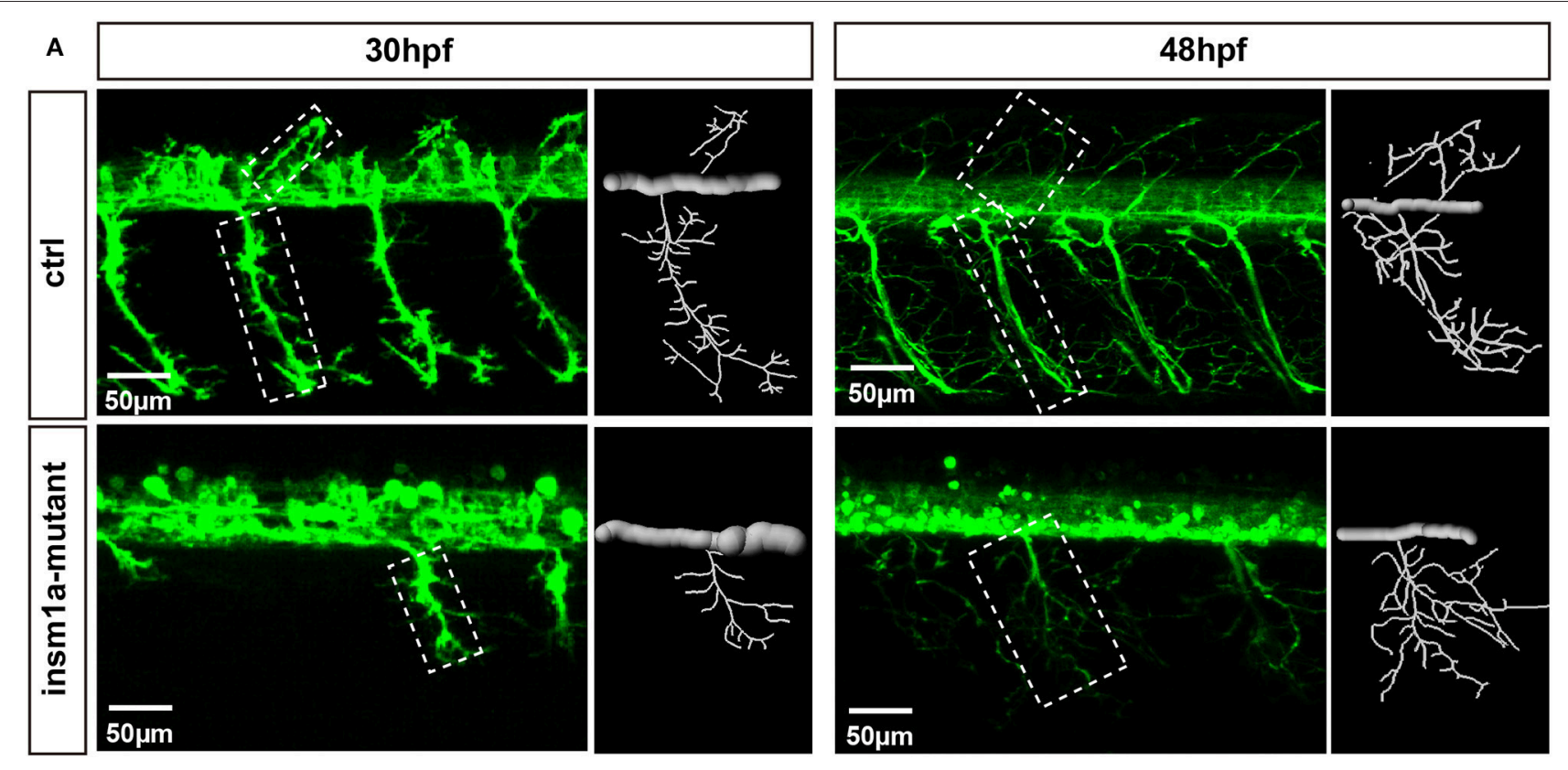

B

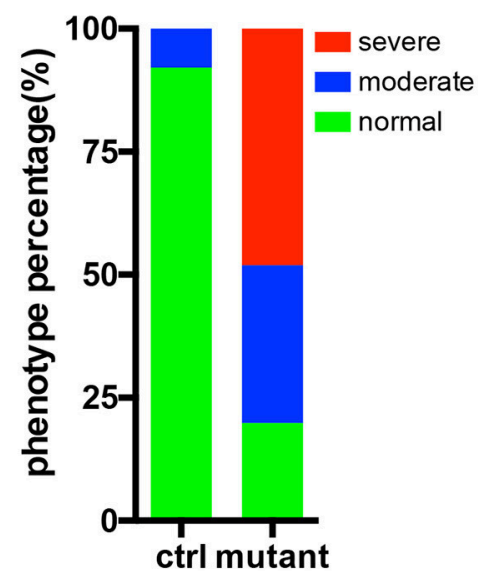

D

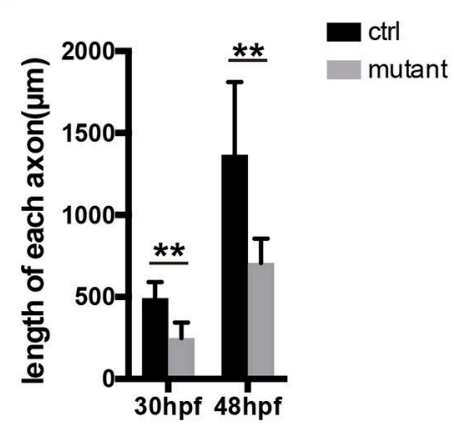

C

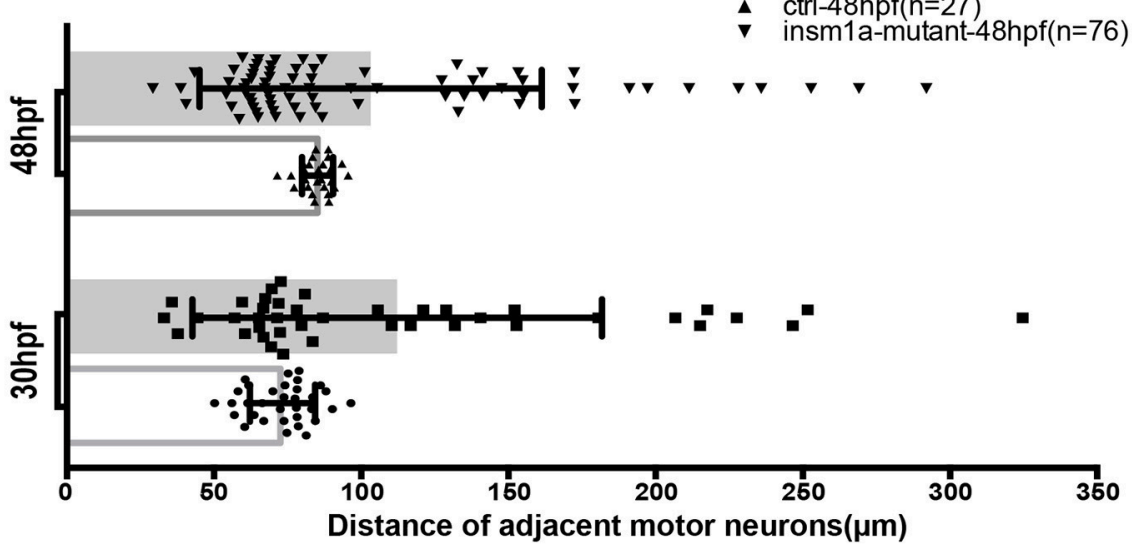

E

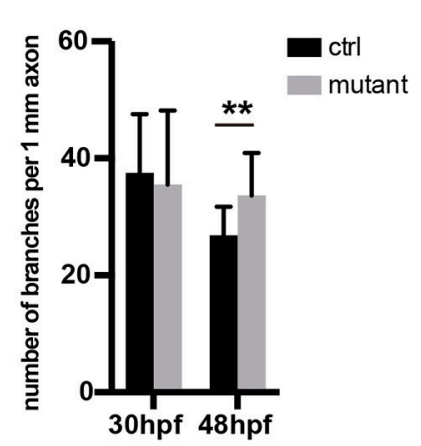

F

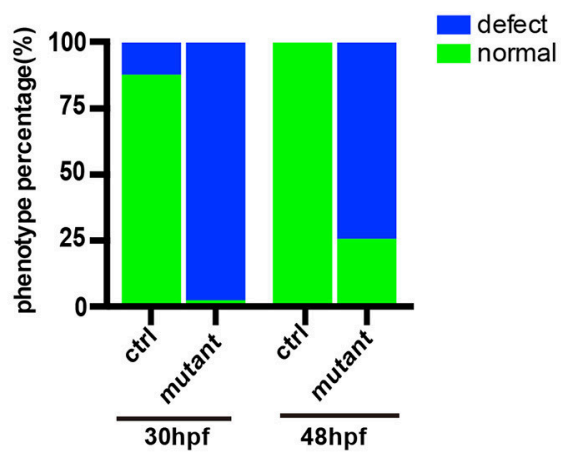

FIGURE 2 | Primary motor neuron morphogenesis defects in the insm1a mutant zebrafish embryos. (A) Confocal imaging analysis of primary motor neuron in control group and insm1a mutant groups at $30 \mathrm{hpf}$ and $48 \mathrm{hpf} \operatorname{Tg}(\mathrm{mn} \times 1 \text { :GFP })^{\mathrm{m} / 2}$. Caps in dash line are showed in diagrams. Scale bar $=50 \mu \mathrm{m}$. (B) Quantification of zebrafish embryos with abnormal Caps. The zebrafish embryos are classified into three categories according to its loss degree: severe group with over $80 \%$ loss of 


\section{FIGURE 2 | Continued}

Cap primary motor neuron, moderate group with $<80 \%$ loss, and normal group with $<20 \%$ loss. (C) Quantification of distance between adjacent motor neurons ( $\mu$ m) in control group and insm1a mutant groups at $30 \mathrm{hpf}(n=33$ and 41 respectively) and 48 hpf ( $n=27$ and 76 respectively). (D,E) The length and branching number of Cap axons in control group and insm1a mutant groups at 30 and 48 hpf. Asterisks above the bars indicate significant differences ( $\left.{ }^{\star \star} P<0.01\right)$. (F) Quantification of zebrafish embryos with abnormal Caps at 30 and $48 \mathrm{hpf}$

insm1a MO into zebrafish embryos, and the results showed that the co-injection significantly decreased the loss, and premature branching of PMNs (Supplementary Figure 4E). Taken together, these results indicated those motor neuron developmental defects were caused by loss of insm1a.

\section{Insm1a Deficiency Suppressed Neuronal Cells Differentiation}

The confocal imaging analysis discovered that there were a number of round and not well-differentiated GFP positive cells in $T g(m n x 1: G F P)^{m l 2}$ insm1a mutants (Figure 3A). Statistical analysis showed that at 30 and $48 \mathrm{hpf}$ the number of these undifferentiated cell in the insmla deficiency zebrafish was significantly higher than that in the control fish (Figure 3B). We also observed these undifferentiated cells in insm1a morphants, however the number was less than that in mutants (Figures 3A,B).

To further investigate the cellular mechanism underlying the motor neuronal defects in insm1a deficient embryos, we performed confocal time-lapse imaging analysis. It was found that in control embryos the axon of $\mathrm{CaP}$ sprouted from the spinal cord, and extended toward to the ventral muscle (Figure 3C). In control embryos the axon of $\mathrm{CaP}$ started to branch when it passed through the midline, while in insm $1 a$ mutants the axon initiated to branch once it came out from the spinal cord (Figures 3A,C, Supplementary Movies 1,2). In addition, we found that those round GFP positive cells did not develop neuronal projections (Figures 3A,C, Supplementary Movie 1).

\section{Knockout of insm1a Reduced the Zebrafish Swimming Activity}

In order to investigate whether the motor neuron defects affects the motor ability, insm1a mutant zebrafish larvae were further performed for 40-min free-swimming activity test independent of any stimuli at 7 and $10 \mathrm{dpf}$. It demonstrated that the movement trajectory and swimming distance per 5 mins, which could reflect the swimming speed, of insm1a mutant zebrafish larvae were significantly decreased compared to that in the control (Figure 4). The movie in the Supplementary Material showed that swimming behavior could be easily discovered in the control group, while the zebrafish in mutant group kept involuntomotory (Supplementary Movie 3). Additionally, we also discovered that under the stereoscopic microscope the mutant zebrafish became insensitive to the touch stimuli (data not shown).

\section{The insm1a Deficiency Caused Alteration of Gene Expression Involved in Motor Neuron Development}

Since insmla is a transcription factor, we supposed that motor neuron developmental defects in insmla deficient embryos were associated with altered expression of downstream genes of insm1a or the genes participating in the motor neuron development. Based on the previous studies, NNR2a, NNR2 $b$, islet2, Ascila, Asci1b, shh, Ngn2, Nkx6.1, and olig2 were selected to do the qRT-PCR analysis in wild-type (WT) and insm1a deficiency zebrafish embryos (Park et al., 2002; Hutchinson et al., 2007; Davis-Dusenbery et al., 2014; Barreiro-Iglesias et al., 2015). The results showed that expressions of NNR2a, NNR2b, islet 2 , Ascila, and Ascilb were significantly influenced in the insmla deficiency zebrafish compared to the control (Supplementary Figure 5). We also found that the expression of shh was obviously elevated in insm 1a mutants at 19, 24, and $36 \mathrm{hpf}$ (Supplementary Figure 5). Interestingly, olig2 and $n k x 6.1$ transcripts dramatically decreased in insm1a deficient embryos (Figures 5A,B).

\section{Olig2 and $n k x 6.1$ Over Expression Rescued the Motor Neuron Defects in insm1a Deficient Embryos}

As the downregulation of olig2 and $n k x 6.1$ in insm1a loss of function embryos, we reasoned that insmla might bind the transcriptional regulatory elements of these two genes. Based on the JASPAR 2016 database (Mathelier et al., 2016) analysis, we found that both olig 2 and $n k x 6.1$ contained the putative binding sites of Insm1a, suggesting Insm1a directly regulates the expression of olig2, and $n k x 6.1$ during PMNs development. To investigate whether the motor neuronal defects in insm1a deficient embryos were caused by reduced expression of olig2 and $n k x 6.1$, we tried to rescue the phenotype with olig2 and nkx6.1 gain of function in insm1a deficient embryos. It was shown that co-injection both olig2 and $n k x 6.1$ mRNA respectively with insm1a MO significantly reduced the motor neuronal defects caused by loss of insm1a (Figures 5C,D). 69.6\% zebrafish embryos injected with insmla $\mathrm{MO}$ at $48 \mathrm{hpf}$ had the motor neuron developmental defects, while only $42.1 \%$ had the motor neuronal phenotype in the olig2 mRNA and insm1a MO coinjection group (Figure 5D). Similarly, after $n k x 6.1 \mathrm{mRNA}$ and insm 1a MO co-injection, the ratio of motor neuronal phenotype decreased to $38.6 \%$ (Figure 5D).

\section{DISCUSSION}

As one of the most conserved zinc-finger transcriptional factor, insmla plays important roles in various biological processes in vertebrates (Wildner et al., 2008; Jacob et al., 2009; Forbes-Osborne et al., 2013; Osipovich et al., 2014; Jia et al., 2015b; Lorenzen et al., 2015). Previous studies have identified its role in regulating the endocrine cells divisions of the pancreas, the neuroendocrine development, the differentiation of retina progenitors and neurogenesis of nervous system (Gierl et al., 2006; Duggan et al., 2008; Farkas et al., 2008; Jacob et al., 2009; 
A

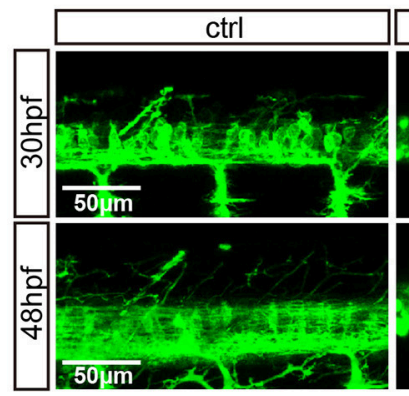

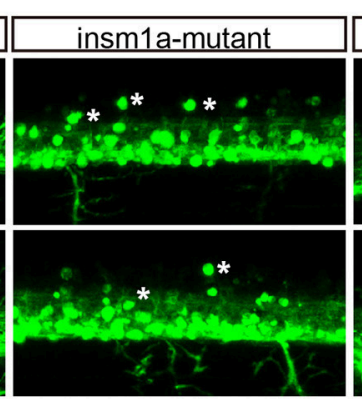

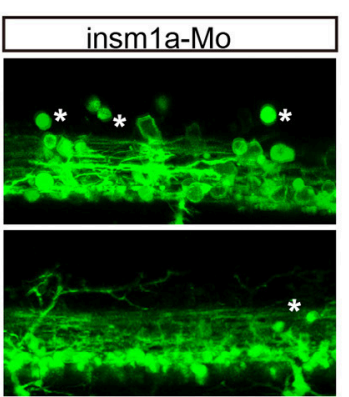

B

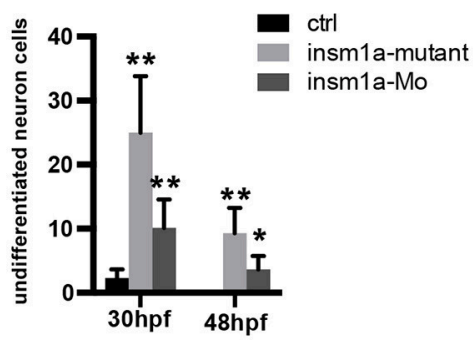

C
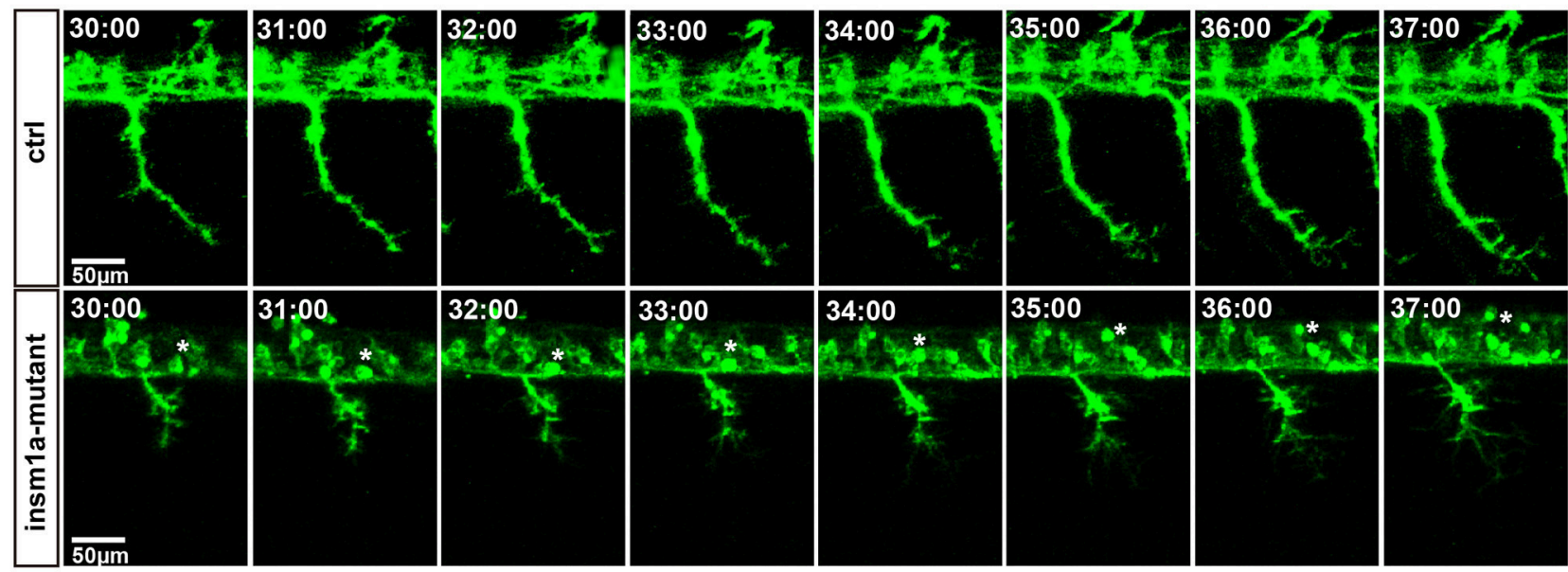

FIGURE 3 | Insm1a deficiency suppressed neuronal cells differentiation. (A) Confocal imaging analysis of primary motor neuron in control group, insm1a mutant group and morphant group at 30 and 48 hpf $\operatorname{Tg}(m n \times 1: G F P)^{m / 2}$. Phenotypes of neuronal cells in the spinal cord in control group, morphant group, and insm1a mutant groups at $30 \mathrm{hpf}$ and $48 \mathrm{hpf}$. Asterisks indicate undifferentiated neuronal cells. Scale bar $=50 \mu \mathrm{m}$. (B) Quantification of the undifferentiated neuronal cell in the insm 1a different treatment zebrafish. Asterisks above the bars indicate significant differences $\left({ }^{\star} P<0.05\right.$, $\left.{ }^{\star \star} P<0.01\right)$. (C) Time-lapse imaging analysis of the primary motor neuron in control group and insm1a mutant groups. Asterisks represent undifferentiated neuronal cells. Scale bar $=50 \mu \mathrm{m}$

Lan and Breslin, 2009; Ramachandran et al., 2012). Currently, our data in this study provided with new insights into the role of insmla in motor neuron development.

Our WISH data and previous study (Ramachandran et al., 2012) demonstrated that insm1a transcripts were detected in retina and spinal cord at $24 \mathrm{hpf}$. Furthermore, imaging analysis of our established transgenic reporter line $\mathrm{Tg}\left(\right.$ insm $1 a: ~ E G F P^{n t u 804}$ and Tg(insm1a: $m$ Cherry) ${ }^{\text {ntu }} 805$ verified the results of in situ hybridization, and revealed the expression of EGFP or mCherry that were driven by insmia promoter in the PMNs. It is wellknown that the spinal cord contains PMNs which project their axons out of the spinal cord to the terminal musculature with the embryo development (Davis-Dusenbery et al., 2014). Taken together, the localization data of insmla from both in situ hybridization analysis and the study based on transgenic reporter line suggested that insm 1a might participate in the regulation of PMNs development.

To test whether insm1a was required for formation of PMNs, we generated CRISPR/Cas9-mediated insm1a mutants, and showed that obvious motor neuron loss and defects of the PMNs axons. Moreover, we also performed insm 1a knockdown, and the results showed similar PMNs defects as the ones produced by the insmla knockout. In wild embryos, exuberant side branches developed at around $72 \mathrm{hpf}$, and then invaded into myotome to form distributed neuromuscular synapses (Liu and Westerfield, 1990; Downes and Granato, 2004). These results suggested that insm1a was pivotal for the primary motor axon development to block precociously extending into muscle territories. Additionally, locomotion analysis displayed a typical low activity swimming behavior in insm1a mutant zebrafish. It was known motor neuron was a major kind of cell type that regulated swimming behavior in zebrafish during early development (Brustein et al., 2003). Previous studies also showed a significant involvement of motor neuron in the overall locomotor behavior (Flanagan-Steet et al., 2005; Levin et al., 2009). Currently, the decrease of swimming activity in this study was consistent with the motor neuron defects in the insmla knockout zebrafish. 
A

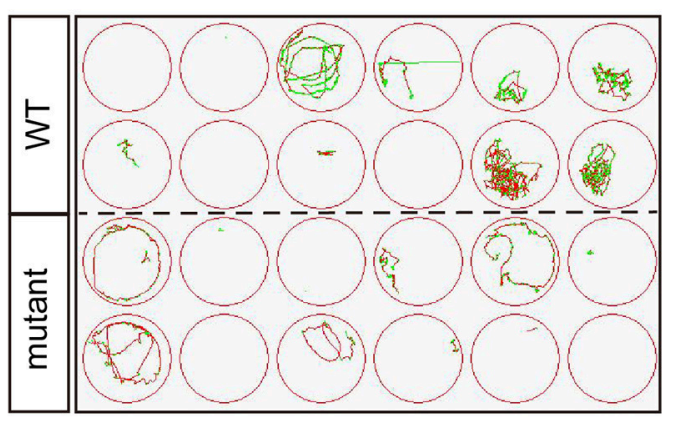

C

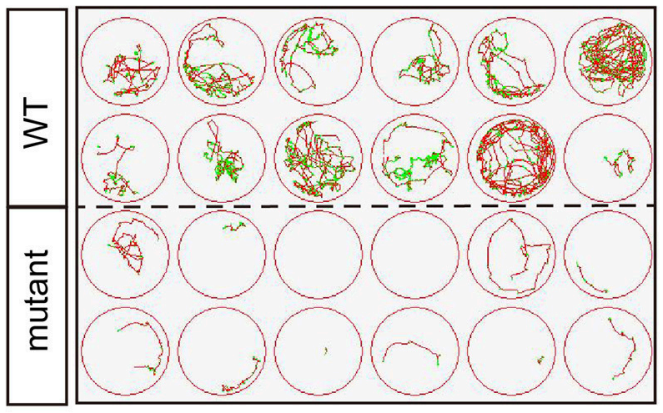

B

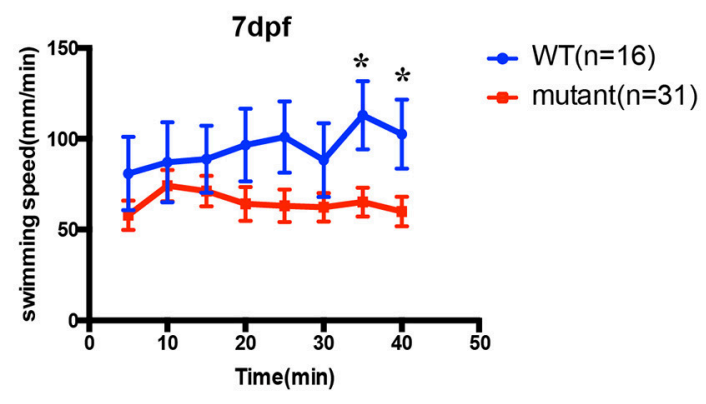

D

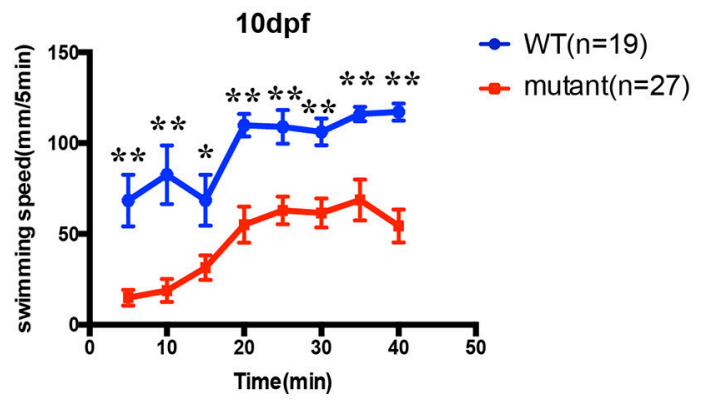

FIGURE 4 | The swimming behavior analysis of control and insm1a mutant zebrafish embryos at 7 and 10 dpf. (A,C). The swimming trajectory of the control and insm1a mutant zebrafish embryos at 7 and $10 \mathrm{dpf}$. (B,D). Quantification of the swimming distance of control and insm1a mutant zebrafish embryos at 7 and 10 dpf per 5 mins ( $n=36$ in each group). Asterisks indicate the significant difference $\left({ }^{\star} P<0.05\right.$, $\left.{ }^{\star \star} P<0.01\right)$.

Another prominent phenotype in the insmla deficiency zebrafish was the disorganized distance between adjacent Caps, which might be caused by the ectopic departure of motor axons from the spinal cord (Palaisa and Granato, 2007). During the zebrafish PMNs development, the three kinds of PMN axons firstly longitudinally migrated toward a segmental spinal cord exit point, and then diverged to individualspecific trajectories (Eisen et al., 1986; Myers et al., 1986). It has been reported that axonal exit sites at the spinal cord might be restricted and conserved (Niederlander and Lumsden, 1996). However, the change of distance between adjacent motor axon and the formation of abnormal axons in our study suggested that the motor axons could form exit points at any positions along entire length of spinal cord in insm 1a mutants. The similar phenotypes were also showed in plexin $A 3$ and semaphorin $3 A$ morphants (Feldner et al., 2007; Palaisa and Granato, 2007; Tanaka et al., 2007). Additionally, Birely et al. reported the phenotype that motor axons departed from the spinal cord at the ectopic points accompanied with defects in slow muscle fiber development (Birely et al., 2005). These studies suggested that the low activity swimming behavior in insmla mutant zebrafish might be involved in the ectopic departure of motor axons from the spinal cord.

In this study we also found that loss function of Insmla obviously impaired the motor neuronal differentiation. Similarly, It was shown that Insmla regulated cell differentiation and migration in zebrafish retinal development and regeneration (Ramachandran et al., 2012; Forbes-Osborne et al., 2013). In vertebrates, Insm1 stimulates cell cycle exit by suppressing expression of cell proliferation related genes and relieving repression of p57kip2, a cyclin kinase inhibitor that along with p27kip1 drives cell cycle exit (Dyer and Cepko, 2001). One consequence of insmla driven cell cycle exit is progenitor differentiation (Ramachandran et al., 2012). The undifferentiated cells in the spinal cord of insm1a mutants confirmed the role of this transcriptional factor in cell differentiation in more cell types.

A series of genes have been identified to contribute to motor neuron formation and development (Park et al., 2002; Cheesman et al., 2004). It has been reported that $n k x 6.1$ and olig2 were dynamically expressed in zebrafih motor neuron and required for motor neuron development. Downregulation of the two genes lead to developmental defect of motor neuron, which was similar with that in insm1a mutant (Park et al., 2002; Cheesman et al., 2004; Hutchinson et al., 2007). Conversely, overexpression of $n k x 6.1$ or olig 2 by mRNA injection could significantly promote the development of the PMNs (Park et al., 2002; Hutchinson et al., 2007). Current study revealed that the inactivation of insm $1 a$ resulted in the significant decrease of $n k x 6.1$ and olig2 expression levels. Furthermore, olig2, and $n k x 6.1$ overexpression rescued the motor neuron defects in insmla deficient embryos. These data suggested that insm 1a regulated the motor neuron development, at least in part, by regulating the expressions of olig2, and $n k x 6.1$. 


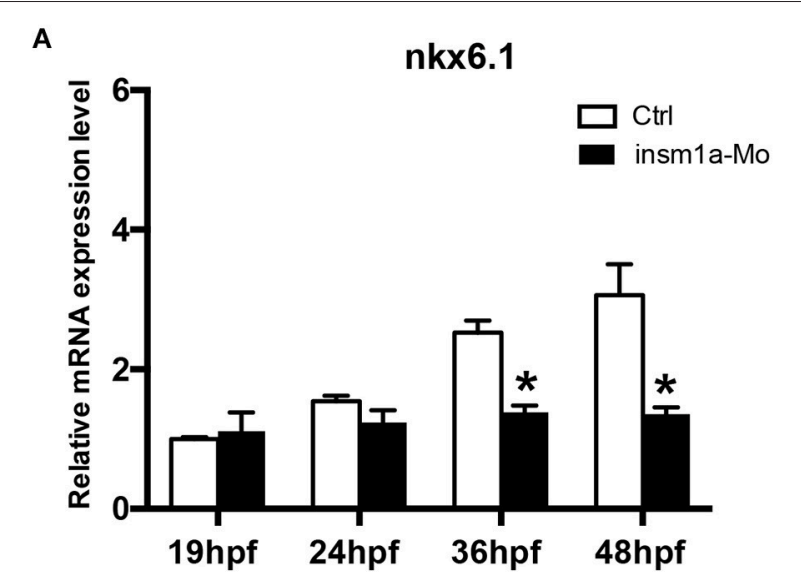

C
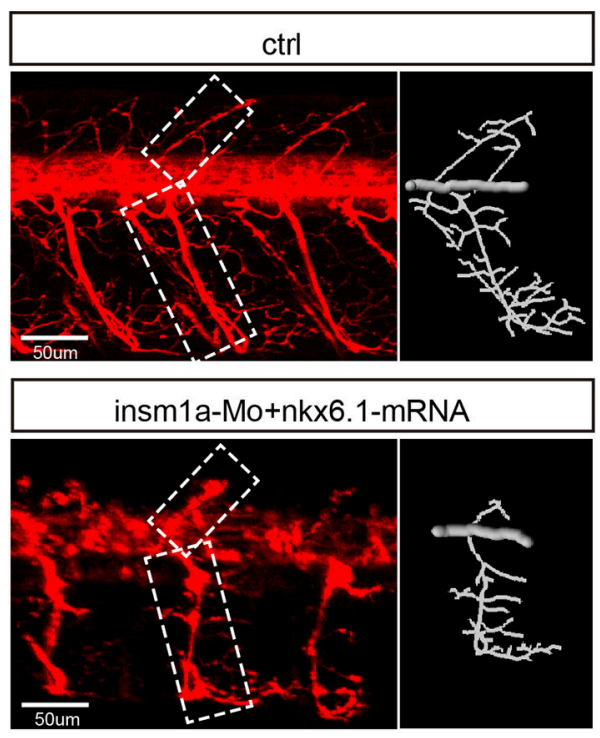
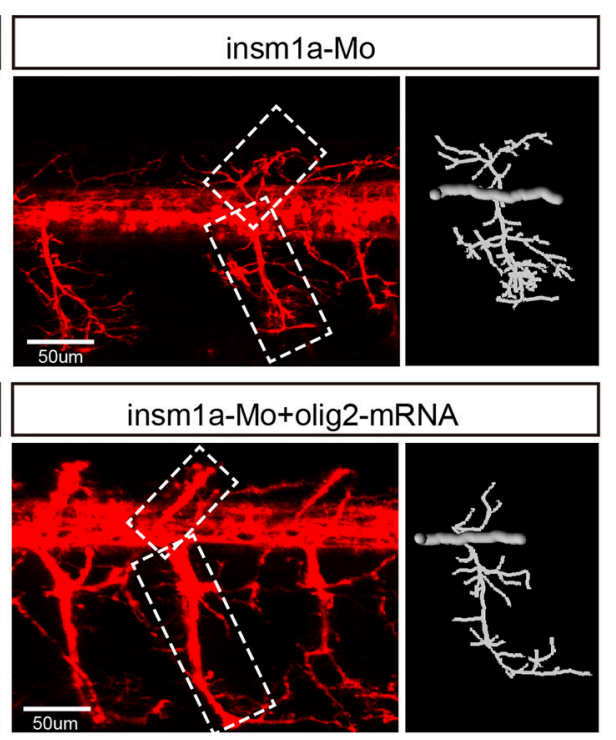

B

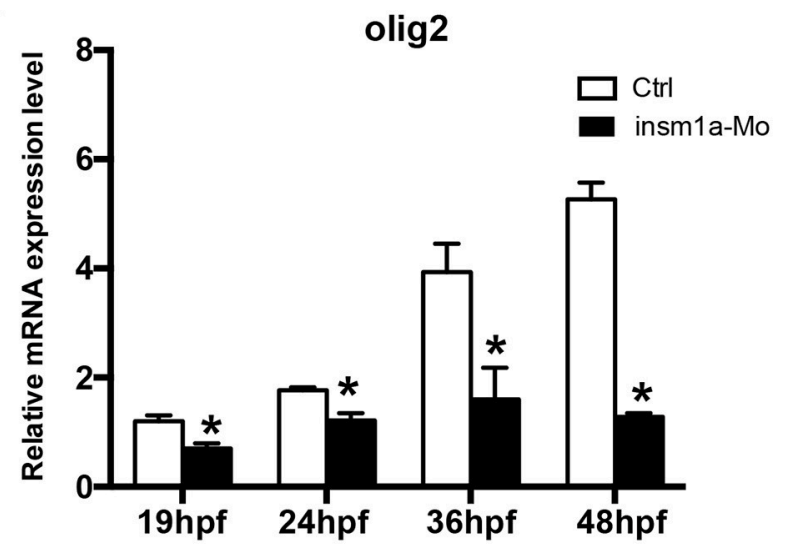

FIGURE 5 | Over expressions of $n k x 6.1$ and olig2 rescued the motor neuron defects in insm1a deficient embryos. (A,B). Effects of insm1a knockdown on the expressions of $n k x 6.1$ and olig2 at 19, 24, 36, and 48 hpf. Asterisks indicate significant differences $\left({ }^{*} P<0.05\right)$. (C) Abnormal Caps in insm1a knockdown zebrafish embryos were restored by co-injection of $n k x 6.1$ or olig2 mRNA. Diagrams of Caps in dash line are displayed near the corresponding confocal image. Scale bar $=50$ $\mu \mathrm{m}$. (D) Quantification of zebrafish embryos with abnormal Cap primary motor neuron. Asterisks indicate significant differences (** $P<0.01)$.

\section{ETHICS STATEMENT}

All animal experimentation was carried out in accordance with the NIH Guidelines for the care and use of laboratory animals (http://oacu.od.nih.gov/regs/index.htm) and ethically approved by the Administration Committee of Experimental Animals, Jiangsu Province, China [Approval ID: SYXK (SU) 2007-0021].

\section{AUTHOR CONTRIBUTIONS}

DL, RC, and QSZ conceived the project. JG, XW, CZ, XNW, $\mathrm{XCD}, \mathrm{FQ}, \mathrm{YS}$, and YG performed most of the experiments. XHD and QXZ generated the mutants. DL, JG, and XW analyzed the data and prepared the manuscript. All authors commented and approved the manuscript.

\section{ACKNOWLEDGMENTS}

This study was supported by grants from the National Natural Science Foundation of China (31400918, 41606169, 81570447, 81622013, 81470692, 31500852); Natural Science Foundation of Jiangsu Province (BK20160418, BK20150022, BK20140620); the National Key Research Development Program of China (2017YFA0103900, 2015CB965000, 2017YFA0103903); the Yingdong Huo Education Foundation; and the Fundamental Research Funds for the Central Universities.

\section{SUPPLEMENTARY MATERIAL}

The Supplementary Material for this article can be found online at: http://journal.frontiersin.org/article/10.3389/fnmol. 2017.00274/full\#supplementary-material 


\section{REFERENCES}

Barreiro-Iglesias, A., Mysiak, K. S., Scott, A. L., Reimer, M. M., Yang, Y., Becker, C. G., et al. (2015). Serotonin promotes development and regeneration of spinal motor neurons in Zebrafish. Cell Rep. 13, 924-932. doi: 10.1016/j.celrep.2015.09.050

Beattie, C. E., Granato, M., and Kuwada, J. Y. (2002). Cellular, genetic and molecular mechanisms of axonal guidance in the zebrafish. Results Probl. Cell Differ. 40, 252-269. doi: 10.1007/978-3-540-46041-1_13

Birely, J., Schneider, V. A., Santana, E., Dosch, R., Wagner, D. S., Mullins, M. C., et al. (2005). Genetic screens for genes controlling motor nerve-muscle development and interactions. Dev. Biol. 280, 162-176. doi: 10.1016/j.ydbio.2005.01.012

Brustein, E., Saint-Amant, L., Buss, R. R., Chong, M., McDearmid, J. R., and Drapeau, P. (2003). Steps during the development of the zebrafish locomotor network. J. Physiol. Paris 97, 77-86. doi: 10.1016/j.jphysparis.2003.10.009

Chang, N., Sun, C., Gao, L., Zhu, D., Xu, X., Zhu, X., et al. (2013). Genome editing with RNA-guided Cas9 nuclease in zebrafish embryos. Cell Res. 23, 465-472. doi: $10.1038 / \mathrm{cr} .2013 .45$

Cheesman, S. E., Layden, M. J., Von Ohlen, T., Doe, C. Q., and Eisen, J. S. (2004). Zebrafish and fly Nkx6 proteins have similar CNS expression patterns and regulate motoneuron formation. Development 131, 5221-5232. doi: 10.1242/dev.01397

Davis-Dusenbery, B. N., Williams, L. A., Klim, J. R., and Eggan, K. (2014). How to make spinal motor neurons. Development 141, 491-501. doi: 10.1242/dev.097410

Downes, G. B., and Granato, M. (2004). Acetylcholinesterase function is dispensable for sensory neurite growth but is critical for neuromuscular synapse stability. Dev. Biol. 270, 232-245. doi: 10.1016/j.ydbio.2004.02.027

Duggan, A., Madathany, T., de Castro, S. C., Gerrelli, D., Guddati, K., and GarciaAnoveros, J. (2008). Transient expression of the conserved zinc finger gene INSM1 in progenitors and nascent neurons throughout embryonic and adult neurogenesis. J. Comp. Neurol. 507, 1497-1520. doi: 10.1002/cne.21629

Dyer, M. A., and Cepko, C. L. (2001). p2 $7^{\mathrm{Kip} 1}$ and $\mathrm{p} 57^{\mathrm{Kip} 2}$ regulate proliferation in distinct retinal progenitor cell populations. J. Neurosci. 21, 4259-4271. Available online at: http://www.jneurosci.org/content/21/12/4259.long

Eisen, J. S. (1991). Determination of primary motoneuron identity in developing zebrafish embryos. Science 252, 569-572. doi: 10.1126/science.1708527

Eisen, J. S., Myers, P. Z., and Westerfield, M. (1986). Pathway selection by growth cones of identified motoneurones in live zebra fish embryos. Nature 320, 269-271. doi: 10.1038/320269a0

Farel, P. B., and Bemelmans, S. E. (1985). Specificity of motoneuron projection patterns during development of the bullfrog tadpole (Rana catesbeiana). J. Comp. Neurol. 238, 128-134. doi: 10.1002/cne.902380112

Farkas, L. M., Haffner, C., Giger, T., Khaitovich, P., Nowick, K., Birchmeier, C., et al. (2008). Insulinoma-associated 1 has a panneurogenic role and promotes the generation and expansion of basal progenitors in the developing mouse neocortex. Neuron 60, 40-55. doi: 10.1016/j.neuron.2008.09.020

Feldner, J., Reimer, M. M., Schweitzer, J., Wendik, B., Meyer, D., Becker, T., et al. (2007). PlexinA3 restricts spinal exit points and branching of trunk motor nerves in embryonic zebrafish. J. Neurosci. 27, 4978-4983. doi: 10.1523/JNEUROSCI.1132-07.2007

Flanagan-Steet, H., Fox, M. A., Meyer, D., and Sanes, J. R. (2005). Neuromuscular synapses can form in vivo by incorporation of initially a neural postsynaptic specializations. Development 132, 4471-4481. doi: 10.1242/dev.02044

Forbes-Osborne, M. A., Wilson, S. G., and Morris, A. C. (2013). Insulinomaassociated 1a (Insm1a) is required for photoreceptor differentiation in the zebrafish retina. Dev. Biol. 380, 157-171. doi: 10.1016/j.ydbio.2013.05.021

Gierl, M. S., Karoulias, N., Wende, H., Strehle, M., and Birchmeier, C. (2006). The zinc-finger factor Insm1 (IA-1) is essential for the development of pancreatic beta cells and intestinal endocrine cells. Genes Dev. 20, 2465-2478. doi: $10.1101 / \operatorname{gad} .381806$

Goto, Y., De Silva, M. G., Toscani, A., Prabhakar, B. S., Notkins, A. L., and Lan, M. S. (1992). A novel human insulinoma-associated cDNA, IA-1, encodes a protein with zinc-finger DNA-binding motifs. J. Biol. Chem. 267, 15252-15257.

Huang, Y., Wang, X., Wang, X., Xu, M., Liu, M., and Liu, D. (2013). Nonmuscle myosin II-B (myh10) expression analysis during zebrafish embryonic development. Gene Expr. Patterns 13, 265-270. doi: 10.1016/j.gep.2013. 04.005

Hutchinson, S. A., Cheesman, S. E., Hale, L. A., Boone, J. Q., and Eisen, J. S. (2007). Nkx6 proteins specify one zebrafish primary motoneuron subtype by regulating late islet1 expression. Development 134, 1671-1677. doi: 10.1242/dev.02826

Jacob, J., Storm, R., Castro, D. S., Milton, C., Pla, P., Guillemot, F., et al. (2009). Insm1 (IA-1) is an essential component of the regulatory network that specifies monoaminergic neuronal phenotypes in the vertebrate hindbrain. Development 136, 2477-2485. doi: 10.1242/dev.034546

Jia, S., Ivanov, A., Blasevic, D., Muller, T., Purfurst, B., Sun, W., et al. (2015a). Insm1 cooperates with Neurod1 and Foxa2 to maintain mature pancreatic beta-cell function. EMBO J. 34, 1417-1433. doi: 10.15252/embj.201490819

Jia, S., Wildner, H., and Birchmeier, C. (2015b). Insm1 controls the differentiation of pulmonary neuroendocrine cells by repressing Hes1. Dev. Biol. 408, 90-98. doi: 10.1016/j.ydbio.2015.10.009

Lan, M. S., and Breslin, M. B. (2009). Structure, expression, and biological function of INSM1 transcription factor in neuroendocrine differentiation. FASEB J. 23, 2024-2033. doi: 10.1096/fj.08-125971

Landmesser, L. T. (1980). The generation of neuromuscular specificity. Annu. Rev. Neurosci. 3, 279-302. doi: 10.1146/annurev.ne.03.030180.001431

Levin, E. D., Aschner, M., Heberlein, U., Ruden, D., Welsh-Bohmer, K. A., Bartlett, S., et al. (2009). Genetic aspects of behavioral neurotoxicology. Neurotoxicology 30, 741-753. doi: 10.1016/j.neuro.2009.07.014

Lewis, K. E., and Eisen, J. S. (2003). From cells to circuits: development of the zebrafish spinal cord. Prog. Neurobiol. 69, 419-449. doi: 10.1016/S0301-0082(03)00052-2

Liu, D. W., and Westerfield, M. (1990). The formation of terminal fields in the absence of competitive interactions among primary motoneurons in the zebrafish. J. Neurosci. 10, 3947-3959.

Lorenzen, S. M., Duggan, A., Osipovich, A. B., Magnuson, M. A., and Garcia-Anoveros, J. (2015). Insm1 promotes neurogenic proliferation in delaminated otic progenitors. Mech. Dev. 138( $\mathrm{Pt} 3), \quad 233-245$. doi: 10.1016/j.mod.2015.11.001

Lukowski, C. M., Ritzel, R. G., and Waskiewicz, A. J. (2006). Expression of two insm1-like genes in the developing zebrafish nervous system. Gene Expr. Patterns 6, 711-718. doi: 10.1016/j.modgep.2005.12.008

Mathelier, A., Fornes, O., Arenillas, D. J., Chen, C. Y., Denay, G., Lee, J., et al. (2016). JASPAR 2016: a major expansion and update of the open-access database of transcription factor binding profiles. Nucleic Acids Res. 44, D110D115. doi: 10.1093/nar/gkv1176

Myers, P. Z. (1985). Spinal motoneurons of the larval zebrafish. J. Comp. Neurol. 236, 555-561. doi: 10.1002/cne.902360411

Myers, P. Z., Eisen, J. S., and Westerfield, M. (1986). Development and axonal outgrowth of identified motoneurons in the zebrafish. J. Neurosci. 6, 2278-2289.

Niederlander, C., and Lumsden, A. (1996). Late emigrating neural crest cells migrate specifically to the exit points of cranial branchiomotor nerves. Development 122, 2367-2374.

Osipovich, A. B., Long, Q., Manduchi, E., Gangula, R., Hipkens, S. B., Schneider, J., et al. (2014). Insml promotes endocrine cell differentiation by modulating the expression of a network of genes that includes Neurog3 and Ripply3. Development 141, 2939-2949. doi: 10.1242/dev.104810

Palaisa, K. A., and Granato, M. (2007). Analysis of zebrafish sidetracked mutants reveals a novel role for Plexin A3 in intraspinal motor axon guidance. Development 134, 3251-3257. doi: 10.1242/dev.007112

Park, H. C., Mehta, A., Richardson, J. S., and Appel, B. (2002). olig2 is required for zebrafish primary motor neuron and oligodendrocyte development. Dev. Biol. 248, 356-368. doi: 10.1006/dbio.2002.0738

Qi, J., Dong, Z., Shi, Y., Wang, X., Qin, Y., Wang, Y., et al. (2016). NgAgo-based fabp1la gene knockdown causes eye developmental defects in zebrafish. Cell Res. 26, 1349-1352. doi: 10.1038/cr.2016.134

Ramachandran, R., Zhao, X. F., and Goldman, D. (2012). Insmla-mediated gene repression is essential for the formation and differentiation of Muller glia-derived progenitors in the injured retina. Nat. Cell Biol. 14, 1013-1023. doi: $10.1038 /$ ncb2586

Rodino-Klapac, L. R., and Beattie, C. E. (2004). Zebrafish topped is required for ventral motor axon guidance. Dev. Biol. 273, 308-320. doi: 10.1016/j.ydbio.2004.06.007 
Shirasaki, R., and Pfaff, S. L. (2002). Transcriptional codes and the control of neuronal identity. Annu. Rev. Neurosci. 25, 251-281. doi: 10.1146/annurev.neuro.25.112701. 142916

Tanaka, H., Maeda, R., Shoji, W., Wada, H., Masai, I., Shiraki, T., et al. (2007). Novel mutations affecting axon guidance in zebrafish and a role for plexin signalling in the guidance of trigeminal and facial nerve axons. Development 134, 3259-3269. doi: 10.1242/dev.004267

Wang, X., Ling, C. C., Li, L., Qin, Y., Qi, J., Liu, X., et al. (2016). MicroRNA-10a/10b represses a novel target gene mib1 to regulate angiogenesis. Cardiovasc. Res. 110, 140-150. doi: $10.1093 / \mathrm{cvr} / \mathrm{cvw} 023$

Westerfield, M., McMurray, J. V., and Eisen, J. S. (1986). Identified motoneurons and their innervation of axial muscles in the zebrafish. J. Neurosci. 6, 2267-2277.

Wildner, H., Gierl, M. S., Strehle, M., Pla, P., and Birchmeier, C. (2008). Insm1 (IA-1) is a crucial component of the transcriptional network that controls differentiation of the sympatho-adrenal lineage. Development 135, 473-481. doi: $10.1242 /$ dev.011783

Xie, J., Cai, T., Zhang, H., Lan, M. S., and Notkins, A. L. (2002). The zinc-finger transcription factor INSM1 is expressed during embryo development and interacts with the Cbl-associated protein. Genomics 80, 54-61. doi: 10.1006/geno.2002.6800

Xu, M., Liu, D., Dong, Z., Wang, X., Wang, X., Liu, Y., et al. (2014). Kinesin-12 influences axonal growth during zebrafish neural development. Cytoskeleton 71, 555-563. doi: $10.1002 / \mathrm{cm} .21193$

Zelenchuk, T. A., and Bruses, J. L. (2011). In vivo labeling of zebrafish motor neurons using an mnxl enhancer and Gal4/UAS. Genesis 49, 546-554. doi: 10.1002/dvg.20766

Conflict of Interest Statement: The authors declare that the research was conducted in the absence of any commercial or financial relationships that could be construed as a potential conflict of interest.

Copyright $\odot 2017$ Gong, Wang, Zhu, Dong, Zhang, Wang, Duan, Qian, Shi, Gao, Zhao, Chai and Liu. This is an open-access article distributed under the terms of the Creative Commons Attribution License (CC BY). The use, distribution or reproduction in other forums is permitted, provided the original author(s) or licensor are credited and that the original publication in this journal is cited, in accordance with accepted academic practice. No use, distribution or reproduction is permitted which does not comply with these terms. 